\title{
On the connectedness of the branch locus of moduli space of hyperelliptic Klein surfaces with one boundary
}

\author{
Antonio F. Costa* \\ Dept. Matemáticas Fundamentales, \\ Facultad de Ciencias, UNED, \\ 28040 Madrid, Spain. \\ Ana M. Porto* \\ Dept. Matemáticas Fundamentales, \\ Facultad de Ciencias, UNED, \\ 28040 Madrid, Spain.
}

\author{
Milagros Izquierdo* \\ Matematiska Institutionen, \\ Linköpings Universitet, \\ 58183 Linköping, Sweden.
}

To the memory of Mika Seppälä.

2000 Mathematics Suject Classification. Primary 30F10, 30F50; Secondary 14H15, $20 \mathrm{H} 10$.

ABSTRACT. In this work we prove that the hyperelliptic branch locus of orientable Klein surfaces of genus $g$ with one boundary component is connected and in the case of non-orientable Klein surfaces it has $\frac{g+1}{2}$ components, if $g$ is odd, and $\frac{g+2}{2}$ components for even $g$. We notice that, for non-orientable Klein surfaces with two boundary components, the hyperelliptic branch loci are connected for all genera.

\section{Introduction}

Moduli space of Klein surfaces is the set of dianalytic structures on a given topological compact surface (possibly non-orientable, with boundary), and it has a natural topology given as quotient of Teichmüller space. The aim of this work is a better understanding of some topological properties of moduli

*Partially supported by MTM2014-55812 
spaces. Connectedness is specially important for subsets of moduli spaces because it allows the deformation of structures with given types.

F. Klein conjectured, and M. Seppälä showed, that the set of real Riemann surfaces is a connected subspace of the moduli space [K, Se, CI1]. To prove this result M. Seppälä uses the connectedness of the locus of hyperelliptic real Riemann surfaces.

The moduli space has an orbifold structure whose singular locus consists of surfaces with non-trivial automorphism [MS]. Branch loci of moduli spaces of Riemann surfaces are connected only for few genera [BCI] (see also $[\mathrm{BI}]$ ); this is in contrast to the case of moduli spaces of orientable Klein surfaces whose branch loci are connected [CIP] (see also [BCIP] for the branch loci of moduli spaces of Riemann surfaces considered as Klein surfaces). Bujalance et al. [BEM] have recently shown that the branch loci of moduli spaces of non-orientable surfaces without boundary and low genus is connected (compare with [BI] and [CI2] for the case of Riemann surfaces).

In this work we study the connectedness of the hyperelliptic branch locus of Klein surfaces with one boundary component. We show that the hyperelliptic branch locus of orientable Klein surfaces with one boundary component is connected and we prove that it is disconnected in the nonorientable case. In this last case we characterize the connected components of the hyperelliptic branch locus in terms of topological types of actions of automorphisms.

Finally, we show that the hyperelliptic branch locus for non-orientable Klein surfaces of topological genus 2 with two boundary components is an example of connected branch loci. We observe that, with the same method, one proves the connectedness of the hyperelliptic branch loci of non-orientable Klein surfaces with two boundary components and topological genus $\geq 2$.

\section{Klein surfaces and non-euclidean crystallographic groups}

A Klein surface $X$ is a compact surface (may be non-orientable and with boundary) endowed with a dianalytic structure, that is to say, a class of atlases where the transition maps are analytic or anti-analytic maps of $\mathbb{C}$ (see [AG, N2, N3]). Klein surfaces are important in the study of real algebraic curves $[\mathrm{G}, \mathrm{CG}]$.

The topological type of $X$ is given by $t=(h, \pm, k)$ where $h$ is the genus, + if $X$ is orientable and - if $X$ is non-orientable and $k$ is the number of connected components of the boundary. The integer $\varepsilon h+k-1$, where $\varepsilon=2$ 
if there is a $+\operatorname{sing}$ in $t$ and $\varepsilon=1$ if there is a $-\operatorname{sign}$ in $t$, is the algebraic genus of $X$.

A non-Euclidean crystallographic group or NEC group $\Gamma$ is a discrete subgroup of the group Aut $^{ \pm} \mathbb{D}$ of conformal and anticonformal automorphisms of the unit disc $\mathbb{D}$ of $\mathbb{C}$ and in this paper we shall assume that the orbit space $\mathbb{D} / \Gamma$ is compact. When the NEC group $\Gamma$ does not contain any orientation-reversing automorphism of $\mathbb{D}$, we say that $\Gamma$ is a Fuchsian group.

The algebraic structure of $\Gamma$ and the geometric and topological structures of the quotient orbifold $\mathbb{D} / \Gamma$ are given by the signature:

$$
s(\Gamma)=\left(h ; \pm ;\left[m_{1}, \ldots, m_{r}\right] ;\left\{\left(n_{1,1}, \ldots, n_{1, r_{1}}\right), \ldots,\left(n_{k, 1}, \ldots, n_{k, r_{k}}\right)\right\}\right) .
$$

See $[\mathrm{W}, \mathrm{M}, \mathrm{Si}]$. The orbit space $\mathbb{D} / \Gamma$ is an orbifold with underlying surface of genus $h$, having $r \geq 0$ cone points and $k$ boundary components, each with $r_{i} \geq 0$ corner points, $i=1, \ldots, k$. The signs " + " and " $-"$ correspond to orientable and non-orientable quotient surfaces respectively. The integers $m_{i}$ are called the proper periods of $\Gamma$; they are the orders of the cone points of $\mathbb{D} / \Gamma$. The brackets $\left(n_{i, 1}, \ldots, n_{i, r_{i}}\right)$ are the period cycles of $\Gamma$. The integers $n_{i, j}$ are the link periods of $\Gamma$ : the orders of the corner points of $\mathbb{D} / \Gamma$. The group $\Gamma$ is isomorphic to the fundamental group of the orbifold $\mathbb{D} / \Gamma$.

Given an NEC group $\Gamma$, the subgroup $\Gamma^{+}$consisting of the orientationpreserving elements of $\Gamma$ is called the canonical Fuchsian subgroup of $\Gamma$.

A group $\Gamma$ with signature (1) has a canonical presentation with generators :

1. Hyperbolic generators: $a_{1}, b_{1}, \ldots, a_{h}, b_{h}$ if $\mathbb{D} / \Gamma$ is orientable; or glide reflection generators: $d_{1}, \ldots, d_{h}$ if $\mathbb{D} / \Gamma$ is non-orientable,

2. Elliptic generators: $x_{1}, \ldots, x_{r}$,

3. Connecting generators (hyperbolic or elliptic transformations): $e_{1}, \ldots, e_{k}$

4. Reflection generators: $c_{i, j}, 1 \leq i \leq k, 1 \leq j \leq r_{i}+1$.

And relators:

1. $x_{i}^{m_{i}}, i=1, \ldots, r$,

2. $c_{i, j}^{2}$,

3. $\left(c_{i, j-1} c_{i, j}\right)^{n_{i, j}}, j=1, \ldots, r_{i}$,

4. $e_{i}^{-1} c_{i, r_{i}} e_{i}^{-1} c_{i, 0}, i=1, \ldots, k$,

5. The long relation, either

$x_{1} \ldots x_{r} e_{1} \ldots e_{k} a_{1} b_{1} a_{1}^{-1} b_{1}^{-1} \ldots a_{h} b_{h} a_{h}^{-1} b_{h}^{-1}$ or $x_{1} \ldots x_{r} e_{1} \ldots e_{k} d_{1}^{2} \ldots d_{h}^{2}$,

according to whether $\mathbb{D} / \Gamma$ is orientable or not.

The hyperbolic area of the orbifold $\mathbb{D} / \Gamma$ coincides with the hyperbolic area of an arbitrary fundamental region of $\Gamma$ and it equals:

$$
\mu(\Gamma)=2 \pi\left(\varepsilon h-2+k+\sum_{i=1}^{r}\left(1-\frac{1}{m_{i}}\right)+\frac{1}{2} \sum_{i=1}^{k} \sum_{j=1}^{r_{i}}\left(1-\frac{1}{n_{i, j}}\right),\right.
$$


where $\varepsilon=2$ if there is a ${ }^{\prime \prime}+{ }^{\prime \prime} \operatorname{sign}$ and $\varepsilon=1$ otherwise. If $\Gamma^{\prime}$ is a subgroup of $\Gamma$ of finite index then $\Gamma^{\prime}$ is an NEC group and the following Riemann-Hurwitz formula holds:

$$
\left[\Gamma: \Gamma^{\prime}\right]=\mu\left(\Gamma^{\prime}\right) / \mu(\Gamma) .
$$

An NEC (Fuchsian) group $\Gamma$ without elliptic elements is called a surface NEC (Fuchsian) group and it has signature $(h ; \pm ;[-],\{(-), . k .,(-)\})$. Any Klein surface $X$ with algebraic genus $\geq 2$ can be represented as the orbit space $X=\mathbb{D} / \Gamma$, with $\Gamma$ a surface NEC group. If a finite group $G$ is isomorphic to a group of automorphisms of $X$ then there exists an NEC group $\Delta$ and an epimorphism $\theta: \Delta \rightarrow G$ with $\operatorname{ker}(\theta)=\Gamma$. The NEC group $\Delta$ is the lifting of $G$ to the universal covering $\mathbb{D} \rightarrow \mathbb{D} / \Gamma$.

\section{Topological classification of automorphisms of Klein surfaces}

Two automorphisms $f$ and $g$ of a Klein surface $X$ are topologically equivalent if $f$ and $g$ are conjugated by a homeomorphism of $X$. The topological types of automorphisms are the topological equivalence classes. The topological types of automorphisms are described using topological invariants (see $[\mathrm{BCNS}],[\mathrm{Y}]$ and $[\mathrm{C}]$ ). Here we present the topological types of automorphisms of Klein surfaces of primer order.

Assume that $X$ is a Klein surface with algebraic genus $\geq 2$, and let $\varphi: X \rightarrow X$ be an order $p$ automorphism; where $p>2$ is a prime. The topological type of $\varphi$ is given by the rotation indices of the fixed points of $\varphi$ and the rotation angles of setwise invariant boundary components (note that in case of non-orientable surfaces the rotation angles are defined up sign). If $\varphi$ has $r$ fixed points and leaves setwise invariant $s$ boundary components, the topological type of $\varphi$ is described by the following data $\theta=\left(p ;\left\{n_{1}, \ldots, n_{r}\right\},\left\{m_{1}, \ldots, m_{s}\right\}\right)$ where $1 \leq n_{i}, m_{i} \leq p-1$. Observe that in the case of non-orientable surfaces the data $n_{i}$ and $p-n_{i}$, and $m_{i}$ and $p-m_{i}$ define topologically equivalent automorphisms. The number $n_{i}$ (respectively $m_{i}$ ) means that there is a fixed point of $\varphi$ (respectively a boundary component of $X$ ) where locally $\varphi$ acts topologically as a rotation with angle $2 \pi n_{i} / p$ (resp. $\left.2 \pi m_{i} / p\right)$. The surface $X$ can be uniformized by a group $\Gamma$ with signature $(g ; \pm ;[-],\{(-), . k .,(-)\})$.. The fact of admitting an automorphism of topological type

$$
\theta=\left(p ; \pm ;\left\{n_{1}, \ldots, n_{r}\right\},\left\{m_{1}, \ldots, m_{b}\right\}\right)
$$


implies that there is an NEC group $\Delta$ with signature,

$$
\left(h ; \pm ;[p, \stackrel{r}{.}, p]\left\{(-), \underline{b},(-),(-), \stackrel{\frac{k-b}{p}}{\stackrel{p}{.}},(-)\right\}\right)
$$

an epimorphism $\omega_{\theta}: \Delta \rightarrow C_{p}=\langle\alpha\rangle$ such that $\Gamma=\operatorname{ker} \omega_{\theta}$. If $x_{j}, c_{l}, e_{l}$ are in a set of canonical generators of $\Delta$, must be:

$$
\omega_{\theta}\left(x_{j}\right)=\alpha^{n_{j}}, \omega_{\theta}\left(e_{j}\right)=\alpha^{m_{j}}, \omega_{\theta}\left(e_{w}\right)=1, \text { for } w>b, \omega_{\theta}\left(c_{l}\right)=1
$$

Note that for Riemann-Hurwitz formula $p$ divides $k-b$.

Let $X$ be a Klein surface and let $\iota: X \rightarrow X$ be an involution. The topological invariants for $\iota$, see [BCNS], are mainly related with the set Fix $(\iota)$ of fixed points of $\iota$. The set Fix $(\iota)$ consists of:

(a) a finite number of $r$ isolated points,

(b) a finite number of simple closed curves that we shall call ovals. Ovals will be called twisted or untwisted accordingly to whether they have Möbius band or annular neighbourhoods respectively. Let $q^{+}$be the number of untwisted ovals and $q^{-}$be the number of twisted ones.

(c) a finite number of chains, which we define now. A chain of length $s_{i}$ (we shall consider $s_{i}$ always to be even) is a set $C$ of $s_{i} / 2$ disjoint $\operatorname{arcs}$ properly embedded in $X$ (i. e. the ends of each component of $C$ are in the boundary of $X$ ) such that for each boundary component $B$ of $X$, either $C \cap B=\varnothing$ or $C \cap B$ consists of two distinct points. Chains may also be twisted or untwisted. The natural definition of the to types of chains is obtained by filling the holes of $X$ with discs, see page 462 of [BCNS]. Let $t^{+}$and $t^{-}$be the number of untwisted and twisted chains respectively.

The extra information that we shall need to determine $\iota$ up topological equivalence is

(d) the number $r_{b}$ of setwise fixed boundary components which contain no points of $\operatorname{Fix}(\iota)$

(e) the orientability of $X /\langle\iota\rangle$, where $\langle\iota\rangle$ is the cyclic group of order two generated by $\iota$,

(f) two homological invariants in the case when $\operatorname{Fix}(\iota)=\varnothing$ and that will not be necessary in our work; therefore we will omit them.

All the above information can be presented in a symbol:

$$
\theta=\left(2 ; \pm ; r, r_{b} ; q^{+}, q^{-} ;\left\{s_{1}, \ldots, s_{t^{+}}\right\},\left\{s_{1}, \ldots, s_{t^{-}}\right\}\right)
$$

the sign + is used if $X /\langle\iota\rangle$ is orientable and the - sign is used if $X /\langle\iota\rangle$ is non-orientable (see [BCNS], [N1]). 
By using NEC groups $X$ can be uniformized by a group $\Gamma$ with signature $(g ; \pm ;[-],\{(-), . k .,(-)\})$ and the fact of admitting an involution with topological invariants $\left(2 ; \pm ; r, r_{b} ; q^{+}, q^{-} ;\left\{s_{1}, \ldots, s_{t^{+}}\right\},\left\{s_{1}, \ldots, s_{t^{-}}\right\}\right)$implies that $\Gamma=\operatorname{ker} \omega_{\theta}$ where $\omega_{\theta}: \Delta \rightarrow C_{2}=\langle\iota\rangle, \Delta$ has signature

$$
\left(h ; \pm ;[2, \stackrel{r}{\cdots}, 2]\left\{(-),{ }^{l+r_{b}},(-),(-),{ }^{+}+q^{-},(-),\left(2, s_{1}, 2\right),{ }^{t^{+}}+t^{-},\left(2, s_{t}, 2\right)\right\}\right),
$$

and if, either $a_{i}, b_{i}, x_{i}, e_{j}, c_{i}, c_{i, j}$ or $d_{i}, x_{i}, e_{j}, c_{i}, c_{i, j}$, is a set of canonical generators of $\Delta$, we must have:

$$
\begin{aligned}
\omega_{\theta}\left(x_{i}\right) & =\iota \\
\omega_{\theta}\left(e_{j}\right) & =1 j=1, \ldots, l \text { and } \omega_{\theta}\left(e_{j}\right)=\iota j=l, \ldots, l+r_{b} \\
\omega_{\theta}\left(c_{i}\right) & =1,1 \leq i \leq l+r_{b} \\
\omega_{\theta}\left(e_{j}\right) & =1 j=l+r_{b}+1, \ldots, l+r_{b}+q^{+} \text {and } \\
\omega_{\theta}\left(e_{j}\right) & =\iota j=l+r_{b}+q^{+}+1, \ldots, l+r_{b}+q^{+}+q^{-} \\
\omega_{\theta}\left(c_{i}\right) & =\iota, l+r_{b}+1 \leq i \leq l+r_{b}+q^{+}+q^{-} ; \\
\omega_{\theta}\left(e_{j}\right) & =1 j=l+r_{b}+q^{+}+q^{-}+1, \ldots, l+r_{b}+q^{+}+q^{-}+t^{+} \text {and } \\
\omega_{\theta}\left(e_{j}\right) & =\iota j=l+r_{b}+q^{+}+q^{-}+t^{+}+1, \ldots, l+r_{b}+q^{+}+q^{-}+t^{+}+t^{-} \\
\omega_{\theta}\left(c_{i, j}\right) & =1, \omega_{\theta}\left(c_{i, j+1}\right)=\iota .
\end{aligned}
$$

\section{Moduli spaces}

Let $s$ be a signature of NEC groups (1) and let $\mathcal{G}$ be an abstract group isomorphic to the NEC groups with signature $s$. We denote by $R(s)$ the set of monomorphisms $r: \mathcal{G} \rightarrow \operatorname{Aut}^{ \pm}(\mathbb{D})$ such that $r(\mathcal{G})$ is an NEC group with signature $s$. The set $R(s)$ has a natural topology given by the topology of $\operatorname{Aut}^{ \pm}(\mathbb{D})$. Two elements $r_{1}$ and $r_{2} \in R(s)$ are said to be equivalent, $r_{1} \sim r_{2}$, if there exists $g \in \operatorname{Aut}^{ \pm}(\mathbb{D})$ such that for each $\gamma \in \mathcal{G}, r_{1}(\gamma)=g r_{2}(\gamma) g^{-1}$. The space of classes $\mathbf{T}(s)=R(s) / \sim$ is called the Teichmüller space of NEC groups with signature $s$ (see $[\mathrm{MS}]$ ). The Teichmüller space $\mathbf{T}(s)$ is homeomorphic to $\mathbb{R}^{d(s)}$ where

$$
d(s)=3(\varepsilon h-1+k)-3+\left(2 \sum_{i=1}^{r} m_{i}+\sum_{i=1}^{k} \sum_{j=1}^{s_{i}} n_{i j}\right) .
$$

The modular group $\operatorname{Mod}(\mathcal{G})$ of $\mathcal{G}$ is the quotient $\operatorname{Mod}(\mathcal{G})=\operatorname{Aut}(\mathcal{G}) / \operatorname{Inn}(\mathcal{G})$, where $\operatorname{Inn}(\mathcal{G})$ denotes the inner automorphisms of $\mathcal{G}$. The moduli space for NEC groups with signature $s$ is the quotient $\mathcal{M}_{s}=\mathbf{T}(s) / \operatorname{Mod}(\mathcal{G})$ endowed 
with the quotient topology and we shall denote $\pi_{s}: \mathbf{T}(s) \rightarrow \mathcal{M}_{s}$. Hence $\mathcal{M}_{s}$ is an orbifold with orbifold fundamental $\operatorname{group} \operatorname{Mod}(\mathcal{G})$.

If $s$ is the signature of a surface group uniformizing surfaces of topological type $t$, then we denote $\mathbf{T}(s)$ by $\mathbf{T}_{t}$, and $\mathcal{M}_{s}$ by $\mathcal{M}_{t}$, the Teichmüller and the moduli space of Klein surfaces of topological type $t$.

Let $\mathcal{G}$ and $\mathcal{G}^{\prime}$ be groups isomorphic to NEC groups with signatures $s$ and $s^{\prime}$ respectively. The inclusion mapping $\alpha: \mathcal{G} \rightarrow \mathcal{G}^{\prime}$ induces an embedding $\mathbf{T}(\alpha): \mathbf{T}\left(s^{\prime}\right) \rightarrow \mathbf{T}(s)$ defined by $[r] \mapsto[r \circ \alpha]$. See $[\mathrm{MS}]$.

If a finite group $G$ is isomorphic to a group of automorphisms of Klein surfaces with topological type $t=(g, \pm, k)$, then the action of $G$ is determined by an epimorphism $\theta: \mathcal{D} \rightarrow G$, where $\mathcal{D}$ is an abstract group isomorphic to NEC groups with a given signature $s$ and $\operatorname{ker}(\theta)$ is isomorphic to an abstract surface group $\mathcal{G}$ of signature $(g ; \pm ;[-] ;\{(-), \ldots,(-)\})$. Then there is an inclusion $\alpha: \mathcal{G} \rightarrow \mathcal{D}$ and an embedding $\mathbf{T}(\alpha): \mathbf{T}(s) \rightarrow \mathbf{T}_{t}$. The continuous map $\mathbf{T}(\alpha)$ induces a continuous map $\mathcal{M}_{s} \rightarrow \mathcal{M}_{t}$. Therefore the set $\mathcal{B}_{t}^{G, \theta}$ of points in $\mathcal{M}_{t}$ corresponding to surfaces having a group of automorphisms isomorphic to $G$ and with a fixed action $\theta$ is connected.

\section{Non-orientable Klein surfaces with one bound- ary component}

A Klein surface $X$ of genus $g$ is said to be hyperelliptic if there is an involution $\varphi$ of $X$ such that $X /\langle\varphi\rangle$ has algebraic genus 0 .

The hyperelliptic branch locus $\mathcal{B}_{(g,-, 1)}^{H y p, G, \theta}$ is the set $\mathcal{B}_{(g,-, 1)}^{K, H y p}=\cup_{G, \theta} \mathcal{B}_{(g,-, 1)}^{H y p, G, \theta}$ of points in $\mathcal{M}_{(g,-, 1)}$ corresponding to hyperelliptic surfaces having automorphisms different from the hyperelliptic involution and the identity.

We are interested in the connectedness of $\mathcal{B}_{(g,-, 1)}^{K, H y p}$.

By the last section, the set $\mathcal{B}_{(g,-, 1)}^{H y p, G, \theta}$ of points in $\mathcal{M}_{(g,-, 1)}$ corresponding to hyperelliptic surfaces with a fixed action $\theta$ of a group of automorphisms isomorphic to $G \supsetneq\langle\varphi\rangle$, where $\varphi$ is the hyperelliptic involution, is connected.

Theorem $1 \mathcal{B}_{(g,-, 1)}^{K, \text { Hyp }}$ is disconnected and has $\frac{g}{2}+1$ connected components for $g$ even and $\frac{g+1}{2}$ connected components for $g$ odd.

Proof. A Klein surface $X$ of genus $g$ is said to be hyperelliptic if there is an involution $\varphi$ of $X$ such that $X /\langle\varphi\rangle$ has algebraic genus 0 . When $X$ is non-orientable and with one boundary component the involution $\varphi$ has 
$g$ isolated interior fixed points and an arc of fixed points with ends on the boundary (a chain following [BCNS]).

In terms of uniformization groups, there is an NEC group $\Delta$ with signature $\left(0 ;\left[2^{g}\right] ;\{(2,2)\}\right)$ and an index two surface subgroup $\Gamma$ of $\Delta$ uniformizing $X$.

We have:

$$
\mathcal{B}_{(g,-, 1)}^{K, H y p}=\cup \mathcal{B}_{(g,-, 1)}^{H y p, G, \theta}=\pi_{t}\left(\cup_{s \prec\left(0 ;\left[2^{g}\right] ;\{(2,2)\}\right), \theta} i_{*}\left(\mathbf{T}_{s}\right)\right),
$$

where $s \prec\left(0 ;\left[2^{g}\right] ;\{(2,2)\}\right)$ means signatures of NEC groups containing groups with signature $\left(0 ;\left[2^{g}\right] ;\{(2,2)\}\right)$ and $\theta: \mathcal{D} \rightarrow G$ are epimorphisms such that $\operatorname{ker} \theta$ is isomorphic to the groups with signature $(g ;-;[-] ;\{(-)\})$ and $\mathcal{D}$ is an abstract group isomorphic to NEC groups with signature $s$.

By Theorem 6.3.3 in [BEGG], a group $G$ of automorphisms of a hyperelliptic non-orientable Klein surface $X$ with one boundary component and automorphisms different from the hyperelliptic involution is $C_{2} \times C_{2}$.

Let us give now a geometrical reason of the above fact. The quotient orbifold $X /\langle\varphi\rangle$ is a disc with $g$ conic points of order 2 , two corner points of angle $\pi / 2$ dividing the topological boundary of the disc in two arcs: one arc consisting of points with non-trivial isotropy groups (the projection of the chain); the other arc corresponds to the projection of the boundary component of $X$. Since $\varphi$ is central in $\operatorname{Aut}(X)$, then $\operatorname{Aut}(X) /\langle\varphi\rangle$ acts on the orbifold $X /\langle\varphi\rangle$; thus each arc in the topological boundary of $X /\langle\varphi\rangle$ must be preserved. Since the arcs admit only actions of $C_{2}$, then $\operatorname{Aut}(X) /\langle\varphi\rangle \cong C_{2}$ and $\operatorname{Aut}(X) \cong C_{2} \times C_{2}$.

The possible signatures of NEC groups $\Lambda$ containing the group $\Delta$ as a subgroup of order two are:

$$
\left(0 ;\left[2^{r}\right] ;\{(2, . . ., 2)\}\right) ; \text { with } 2 r+s=g+3
$$

Given a group $\Lambda$ as above, there is a unique epimorphism $\theta_{r}: \Lambda \rightarrow \Lambda / \Gamma=$ $\operatorname{Aut}(X) \cong C_{2} \times C_{2}=\langle a, b\rangle$ such that $\theta_{r}^{-1}(\langle a\rangle)$ has signature $\left(0 ;\left[2^{g}\right] ;\{(2,2)\}\right)$ (i.e. the element $a$ represents the hyperelliptic involution in $X=\mathbb{D} / \Gamma$ ).

Using a canonical presentacion of $\Lambda$, the epimorphism $\theta_{r}$ is: 


$$
\begin{gathered}
\theta_{r}\left(x_{i}\right)=a, i=1, \ldots, r \\
\theta_{r}(e)=a \text { if } r \equiv 1 \bmod 2 \text { and } \theta_{r}(e)=\text { id if } r \equiv 0 \bmod 2 \\
\theta_{r}\left(c_{0}\right)=a \\
\theta_{r}\left(c_{1}\right)=\text { id } \\
\theta_{r}\left(c_{2}\right)=b \\
\theta_{r}\left(c_{3}\right)=a b \\
\theta_{r}\left(c_{4}\right)=b \\
\theta_{r}\left(c_{5}\right)=a b \\
\ldots \\
\theta_{r}\left(c_{s-1}\right)=b, \text { if } s \equiv 1 \bmod 2 \text { and } \theta_{r}\left(c_{s-1}\right)=a b, \text { if } s \equiv 0 \bmod 2 \\
\theta_{r}\left(c_{s}\right)=a
\end{gathered}
$$

For each signature we have an action of $C_{2} \times C_{2}$. Since there are no bigger groups of automorphisms each epimorphism or action is maximal and defines a connected component of $\mathcal{B}_{(g,-, 1)}^{K, H y p}$.

In the next result we describe the topological type of the automorphisms of Klein surfaces in each component of the hyperelliptic branch locus $\mathcal{B}_{(g,-, 1)}^{K, \text { Hyp }}$.

Proposition 2 Let $\pi_{(g,-, 1)} \circ i_{*}\left(\mathbf{T}_{\left(0 ;\left[2^{r}\right] ;\{(2, . s, 2)\}\right)}\right)$ be a connected component of $\mathcal{B}_{(g,-, 1)}^{K, H y p}$. Given $X \in \pi_{(g,-, 1)} \circ i_{*}\left(\mathbf{T}_{\left(0 ;\left[2^{r}\right] ;\{(2, . . ., 2)\}\right)}\right)$, Aut $(X)$ contains:

- the hyperelliptic involution $\varphi$ with g isolated fixed points and one chain, the chain is twisted if $g$ is odd and untwisted if $g$ is even. Following the notation in Section 3, the topological type of the hyperelliptic involution is $(2 ;+; g, 0 ; 0,0 ;\{2\},\{0\})$ if $g$ is even and $(2 ;+; g, 0 ; 0,0 ;\{0\},\{2\})$ if $g$ is odd.

- an involution $\alpha$ with the following topological type: $\left(2 ; \pm ; 1,0 ; \frac{g-1}{2}-\right.$ $r, 0 ;\{0\},\{2\})$ for $g$ odd and $\left(2 ; \pm ; 0,0 ; \frac{g}{2}-r-1,1 ;\{0\},\{2\}\right)$ for $g$ even; that is, the involution $\alpha$ has $\frac{g-1}{2}-r$ untwisted ovals, an isolated fixed point and one twisted chain, for $g$ odd; $\frac{g}{2}-r$ ovals, only one of them twisted (if there are ovals), and one twisted chain, for $g$ even. Finally $X /\langle\alpha\rangle$ is non-orientable if $r>0$ and $X /\langle\alpha\rangle$ is orientable if $r=0$,

- finally, an involution $\alpha \varphi$ with topological type: $\left(2 ;-; 0,0 ; \frac{g+1}{2}-r-\right.$ $2,2 ;\{0\},\{0\})$ for $g$ odd and $\left(2 ;-; 1,0 ; \frac{g}{2}-r-1,1 ;\{0\},\{0\}\right)$ for $g$ even; that is, the involution $\alpha \varphi$ has $\frac{g+1}{2}-r$ ovals, exactly two of them twisted (if there are ovals), for $g$ odd; but $\frac{g}{2}-r$ ovals, one of them twisted (if there are ovals) and one isolated fixed point, for $g$ even. The quotient $X /\langle\alpha \varphi\rangle$ is always non-orientable. 
Proof. Let $X \in \pi_{(g,-, 1)} \circ i_{*}\left(\mathbf{T}_{\left(0 ;\left[2^{r}\right] ;\{(2, s, 2)\}\right)}\right)$. Consider the monodromy $\theta_{r}: \Lambda \rightarrow \Lambda / \Gamma=\operatorname{Aut}(X) \cong C_{2} \times C_{2}=\langle a, b\rangle$ defined in the proof of Theorem 1. Where $a$ represents the hyperelliptic involution $\varphi$ and $b$ represents the involution $\alpha$. Appying [BEGG] and [BCNS], we have that:

$\theta_{r}^{-1}(\langle a\rangle)$ has signature $\left(0 ;\left[2^{g}\right] ;\{(2,2)\}\right)$. Now:

- for $g$ odd:

$\theta_{r}^{-1}(\langle b\rangle)$ has signature $\left(r ;-;[2] ;\left\{(-)^{\frac{g-1}{2} \ldots}-r(-)(2,2)\right\}\right)$ with $r>0$ and $\left(0 ;+;[2] ;\left\{(-) \stackrel{\frac{g-1}{2}}{.}(-)(2,2)\right\}\right)$ with $r=0$,

$\theta_{r}^{-1}(\langle a b\rangle)$ has signature $\left(r+1 ;-;[-] ;\left\{(-)^{\frac{g+1}{2} \ldots}-r(-)\right\}\right)$.

- for $g$ even:

$\theta_{r}^{-1}(\langle b\rangle)$ has signature $\left(r ;-;[-] ;\left\{(-)^{\frac{g}{2}-\cdots}(-)(2,2)\right\}\right)$ with $r>0$ and $\left(0 ;+;[2] ;\left\{(-)^{\frac{g-1}{2}} \cdot(-)(2,2)\right\}\right)$ with $r=0$,

$\theta_{r}^{-1}(\langle a b\rangle)$ has signature $\left(r+1 ;-;[2] ;\left\{(-)^{\frac{g}{2}-*}(-)\right\}\right)$.

The twisted property for chains and ovals is determined by the image of the connecting generator of the NEC groups. For instance, for the case $g$ odd, the twisted chain of the involution $\alpha$ is produced by

$$
\theta_{r}\left(c_{0}\right)=a, \theta_{r}\left(c_{1}\right)=\mathrm{id}, \theta_{r}\left(c_{2}\right)=b, \theta_{r}\left(c_{3}\right)=a b
$$

Remark 3 For genus $g=2$ the disconnectedness of the hyperelliptic branch locus of non-orientable Klein surfaces follows from [Ci].

\section{The branch locus for the moduli of hyperellip- tic orientable Klein surfaces with one boundary component}

Now, we shall study the connectedness of the set $\mathcal{B}_{(g,+, 1)}^{K, H y p}$ that consists of the points in $\mathcal{M}_{(g,+, 1)}$ that are hyperelliptic and with automorphisms different from the hyperelliptic involution and the identity.

Proposition 4 The hyperelliptic branch locus $\mathcal{B}_{(g,+, 1)}^{K, H y p}$ is connected. 
Proof. Consider a surface $X$ in the hyperelliptic branch locus with the hyperelliptic involution $\varphi$. The quotient $X /\langle\varphi\rangle$ is a disc with $2 g+1$ conic points of order 2 (see [BEGG]). Let $G$ be the automorphism group of $X$, the quotient group $G /\langle\varphi\rangle$ is cyclic or dihedral, see [BEGG] (observe that $G /\langle\varphi\rangle$ acts on a disc).

Let $\mathcal{B}_{(g,+, 1)}^{H y p, G, \theta}$ be the connected subset of $\mathcal{B}_{(g,+, 1)}^{K, \text { Hyp }}$ given by the surfaces with automorphism group containing the group $G$ (where $G$ contains the hyperelliptic involution) acting in a fixed way given by $\theta$.

We have: $\mathcal{B}_{(g,+, 1)}^{K, H y p}=\cup \mathcal{B}_{(g,+, 1)}^{H y p, G, \theta}=\pi_{(g,+, 1)}\left(\cup_{s \prec\left(0 ;\left[2^{2 g+1}\right] ;\{(-)\}\right), \theta} i_{*}\left(\mathbf{T}_{s}\right)\right)$, where $s \prec\left(0 ;\left[2^{2 g+1}\right] ;\{(-)\}\right)$ means signatures of NEC groups containing groups with signature $\left(0 ;\left[2^{2 g+1}\right] ;\{(-)\}\right)$ and $\theta: \mathcal{D} \rightarrow G$ are epimorphisms such that $\operatorname{ker} \theta$ is isomorphic to the groups with signature $(g ;+;[-] ;\{(-)\})$ and $\mathcal{D}$ is an abstract group isomorphic to NEC groups with signature $s$. Let $\mathcal{B}(s)=\pi_{(g,+, 1)}\left(\cup_{\theta} i_{*}\left(\mathbf{T}_{s}\right)\right)$, where $s \prec\left(0 ;\left[2^{2 g+1}\right] ;\{(-)\}\right)$ and $\theta$ runs over all epimorphism from groups with signature $s$.

Thus

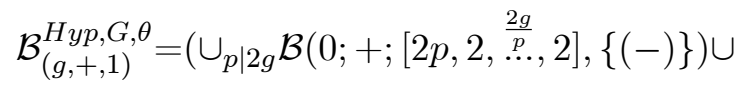

$$
\begin{aligned}
& \cup\left(\cup_{p \mid 2 g+1} \mathcal{B}\left(0 ;+;\left[p, 2, \stackrel{\frac{2 g+1}{\underline{p}}}{\stackrel{p}{*}}, 2\right],\{(-)\}\right) \cup\right. \\
& \left(\cup_{2 r+s=2 g+3} \mathcal{B}(0 ;+;[2, . . ., 2]\{(2, . . ., 2)\}) .\right.
\end{aligned}
$$

The following unique monodromy $\theta_{p}: \Delta \rightarrow D_{p} \times C_{2}=\langle s, t\rangle \times\langle a\rangle$, where $\Delta$ has signature $\left(0 ;+;\left[2, \frac{2 g+1-p}{2 p}, 2\right],\{(p, 2,2,2)\}\right)$ and $\theta_{p}$ is defined by:

$$
x_{i} \rightarrow a
$$

$e \rightarrow a$ or id (according to the parity of $g / p$ )

$$
c_{0} \rightarrow s, c_{1} \rightarrow t, c_{2} \rightarrow t a, c_{3} \rightarrow \mathrm{id}, c_{4} \rightarrow s
$$

yields that

$$
\begin{gathered}
\mathcal{B}\left(0 ;+;\left[2, \frac{\frac{2 g+1-p}{2 p}}{\cdots}, 2\right],\{(p, 2,2,2)\}\right)=\mathcal{B}_{(g,+, 1)}^{H y p, D_{p} \times C_{2}, \theta_{p}} \subset \\
\mathcal{B}\left(0 ;+;\left[p, 2, \stackrel{\frac{2 g+1}{p}}{\stackrel{p}{*}}, 2\right],\{(-)\}\right) \cap \mathcal{B}(0 ;+;[2, \stackrel{g}{.}, 2],\{(2,2,2)\}) \neq \varnothing
\end{gathered}
$$

(the hyperelliptic involution of $\mathbb{D} / \operatorname{ker} \theta_{p}$ is represented by $a$ ). Similarly:

$$
\begin{aligned}
& \mathcal{B}\left(0 ;+;\left[2, \stackrel{\frac{g}{p}}{.}, 2\right],\{(2 p, 2,2)\}\right)=\mathcal{B}_{(g,+, 1)}^{H y p, D_{2 p}, \theta_{p}^{\prime}} \subset \\
& \mathcal{B}\left(0 ;+;\left[2 p, 2, \stackrel{\frac{2 g}{p}}{\underline{p}}, 2\right],\{(-)\}\right) \cap \mathcal{B}(0 ;+;[2, . g, 2],\{(2,2,2)\}) \neq \varnothing \text {. }
\end{aligned}
$$


Now, for the points in $\mathcal{B}_{(g,+, 1)}^{K, H y p}$ having anticonformal involutions we consider the following monodromies:

1. The monodromy $\theta_{-3}: \Delta \rightarrow D_{4}=\langle s, t\rangle$, where $\Delta$ has signature $\left(0 ;+;[2, \ldots, 2],\left\{\left(4,2,{ }^{g+2-2 k}, 2\right)\right\}\right)$, defined by,

$$
\begin{gathered}
x_{i} \rightarrow(s t)^{2} \\
\left.e \rightarrow(s t)^{2} \text { or id (according to the parity of } k\right) \\
c_{0} \rightarrow s \\
c_{1} \rightarrow t \\
c_{2} \rightarrow t(s t)^{2} \\
c_{3} \rightarrow t \\
c_{4} \rightarrow t(s t)^{2} \\
c_{5} \rightarrow t \\
\quad \ldots \\
c_{g+1-2 k} \rightarrow t \text { or } t(s t)^{2} \\
c_{g+2-2 k} \rightarrow \text { id } \\
c_{g+3-2 k} \rightarrow s
\end{gathered}
$$

(the hyperelliptic involution $\varphi$ of $\mathbb{D} / \operatorname{ker} \theta_{-3}$ is represented by $(s t)^{2}$ ).

This yields:

$\mathcal{B}_{(g,+, 1)}^{H y p, D_{4}, \theta_{-3}} \subset \mathcal{B}\left(0 ;+;[2,2 k, 2],\left\{\left(2,{ }^{2 g+3-. .4 k}, 2\right)\right\}\right) \cap \mathcal{B}(0 ;+;[2, . g ., 2],\{(2,2,2)\}) \neq \varnothing$

2. The monodromy $\theta_{-5}: \Delta \rightarrow D_{4}=\langle s, t\rangle$, where $\Delta$ has signature $\left(0 ;+;[2, \ldots, 2],\left\{\left(4,2,{ }^{g+2-2 k}, 2\right)\right\}\right)$, defined by

$$
\begin{aligned}
& x_{i} \rightarrow(s t)^{2} \\
& e \rightarrow(s t)^{2} \text { or id (according to the parity of } k \text { ) } \\
& c_{0} \rightarrow s \\
& c_{1} \rightarrow t \\
& c_{2} \rightarrow t(s t)^{2} \\
& c_{3} \rightarrow t \\
& c_{4} \rightarrow t(s t)^{2} \\
& c_{5} \rightarrow t \\
& c_{g-1-2 k} \rightarrow t \text { or } t(s t)^{2} \\
& c_{g-2 k} \rightarrow \mathrm{id} \\
& c_{g+1-2 k} \rightarrow s \\
& c_{g+2-2 k} \rightarrow t \\
& c_{g+3-2 k} \rightarrow s
\end{aligned}
$$


yields:

$$
\begin{gathered}
\mathcal{B}_{(g,+, 1)}^{H y p, D_{4}, \theta_{-5}} \subset \mathcal{B}\left(0 ;+;[2,2 k, 2],\left\{\left(2,{ }^{2 g+1-}{ }^{-4 k}, 2\right)\right\}\right) \cap \\
\cap \mathcal{B}\left(0 ;+;\left[2,{ }^{g-1}, 2\right],\{(2,2,2,2,2)\}\right) \neq \varnothing
\end{gathered}
$$

3. Finally the monodromy $\theta_{-3,-5}: \Delta \rightarrow D_{4 g}=\langle s, t\rangle$ where $\Delta$ has signature $(0 ;+;[-],\{(4 g, 2,2,2)\})$ defined by:

$$
c_{0} \rightarrow s, c_{1} \rightarrow t, c_{2} \rightarrow \mathrm{id}, c_{3} \rightarrow s(s t)^{2 g}
$$

( $\varphi$ is now represented by $(s t)^{2 g}$ ) produces:

$$
\begin{gathered}
\mathcal{B}_{(g,+, 1)}^{\text {Hyp, } D_{4 g}, \theta_{-3},-5}=\mathcal{B}(0 ;+;[-],\{(4 g, 2,2,2)\}) \subset \\
\mathcal{B}(0 ;+;[2, . \stackrel{g}{.}, 2],\{(2,2,2)\}) \cap \mathcal{B}\left(0 ;+;\left[2,{ }_{\cdots}^{g-1}, 2\right],\{(2,2,2,2,2)\}\right) \neq \varnothing
\end{gathered}
$$

Hence $\mathcal{B}_{(g,+, k)}^{K H}$ is connected.

\section{Non-orientable Klein surfaces with two bound- ary components}

In this section we show, as an example, that the hyperelliptic branch locus of non-orientable Klein surfaces with two boundary components is connected. At the end we shall remark that this situation is general for non-orientable Klein surfaces with two boundary components.

Proposition $5 \mathcal{B}_{(2,-, 2)}^{K, H y p}$ is connected

Proof. Let $X$ be a Klein surface in the hyperelliptic branch locus with the hyperelliptic involution $\varphi$. The signature of the NEC groups uniformizing the quotient orbifold $X /\langle\varphi\rangle$ is: $(0 ;[2,2] ;\{(2,2,2,2)\})$. The topological type of $\varphi$ is given by:

$$
(2 ;+; 2,0 ; 0,0 ;\{4\},\{-\})
$$

that is to say: $\varphi$ has two fixed points and an untwisted chain of length 4 .

The automorphisms of $X$ necessarily have order two, since such a automorphism will induce an automorphism of a disc whose boundary is divided by four corner points in four arcs alternately bicoloured (this bicoloration is given by the projection of the boundary components and points in the chain $\varphi)$. 
If $X$ has an automorphism $\psi$ different from $\varphi$ the possible types of actions of groups $\langle\varphi, \psi\rangle$ produce the following signatures of NEC groups $\Gamma$ such that $X /\langle\varphi, \psi\rangle=\mathbb{D} / \Gamma$ with some epimorphism $\theta: \Gamma \rightarrow C_{2} \times C_{2}$ and $\mathbb{D} / \operatorname{ker} \theta=X$ :

1. $(0 ;[2,2] ;\{(2,2)\})$

2. $(0 ;[2] ;\{(2,2,2,2)\})$

3. $(0 ;[-] ;\{(2,2,2,2,2,2)\})$

Let now describe the equivalence classes of epimorphisms $\theta: \Gamma \rightarrow C_{2} \times$ $C_{2}=\langle a, b\rangle$, the element $a$

1. For signature $(0 ;[2,2] ;\{(2,2)\})$ there is only one class:

$$
\theta_{2}: \quad x_{1} \rightarrow a, x_{2} \rightarrow b, e \rightarrow a b, c_{0} \rightarrow a, c_{1} \rightarrow \mathrm{id}, c_{2} \rightarrow a
$$

2. For signature $(0 ;[2] ;\{(2,2,2,2)\})$ there are two classes $\theta_{1}$ and $\theta_{1}^{\prime}$ defined by:

$$
\begin{aligned}
& \theta_{1}: \quad x \rightarrow a, e \rightarrow a, c_{0} \rightarrow a, c_{1} \rightarrow \mathrm{id}, c_{2} \rightarrow a, c_{3} \rightarrow b, c_{4} \rightarrow a \\
& \theta_{1}^{\prime}: \quad x \rightarrow a, e \rightarrow a, c_{0} \rightarrow a, c_{1} \rightarrow \mathrm{id}, c_{2} \rightarrow b, c_{3} \rightarrow \mathrm{id}, c_{4} \rightarrow a
\end{aligned}
$$

For signature $(0 ;[-] ;\{(2,2,2,2,2,2)\})$ there are two classes $\theta_{0}$ and $\theta_{0}^{\prime}$ defined by:

$$
\begin{aligned}
\theta_{0}: & c_{0} \rightarrow a, c_{1} \rightarrow \mathrm{id}, c_{2} \rightarrow a, c_{3} \rightarrow b, c_{4} \rightarrow b a, c_{5} \rightarrow b, c_{6} \rightarrow a \\
\theta_{0}^{\prime}: & c_{0} \rightarrow \mathrm{id}, c_{1} \rightarrow a, c_{2} \rightarrow \mathrm{id}, c_{3} \rightarrow b, c_{4} \rightarrow a b, c_{5} \rightarrow b, c_{6} \rightarrow \mathrm{id}
\end{aligned}
$$

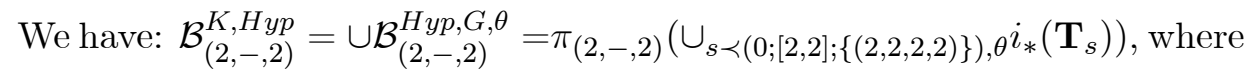
$s \prec(0 ;[2,2] ;\{(2,2,2,2)\})$ means signatures $1,2,3$ above and $\theta$ is $\theta_{i}, i=$ $0,1,2$ or $\theta_{j}^{\prime}, j=0,1$.

We shall denote the connected set $\pi_{(2,-, 2)}\left(\cup_{s \prec(0 ;[2,2] ;\{(2,2,2,2)\}), \theta} i_{*}\left(\mathbf{T}_{s}\right)\right)$ by $\mathcal{B}_{(2,-, 2)}^{\text {Hyp }, \theta}(s)$.

Consider the monodromies $\theta_{0^{\prime}, 1}: \Delta \rightarrow C_{2} \times C_{2} \times C_{2}=\langle b, m, a\rangle$ (the hyperelliptic involution $\varphi$ is represented by $a$ ) and $\theta_{0,1^{\prime}}^{\prime}: \Delta \rightarrow C_{2} \times C_{2} \times C_{2}$, with $\Delta$ of signature $(0 ;[-]\{(2,2,2,2,2)\})$ defined by:

$$
\begin{aligned}
& \theta_{0^{\prime}, 1}: \quad c_{0} \rightarrow m, c_{1} \rightarrow a, c_{2} \rightarrow \mathrm{id}, c_{3} \rightarrow a b, c_{4} \rightarrow b, c_{5} \rightarrow m \\
& \theta_{01^{\prime}}: \quad c_{0} \rightarrow m, c_{1} \rightarrow \mathrm{id}, c_{2} \rightarrow a, c_{3} \rightarrow a b, c_{4} \rightarrow b, c_{5} \rightarrow m
\end{aligned}
$$

We have (see $[$ BEGG]):

$$
\begin{aligned}
& \mathcal{B}_{(2,-, 2)}^{H y p, C_{2} \times C_{2} \times C_{2}, \theta_{0^{\prime} 1}} \subset \mathcal{B}(0 ;[2,2] ;\{(2,2)\}) \cap \mathcal{B}_{(2,-, 2)}^{H y p, C_{2} \times C_{2}, \theta_{1}} \cap \mathcal{B}_{(2,-, 2)}^{H y p, C_{2} \times C_{2}, \theta_{0^{\prime}}} \neq \varnothing \\
& \quad \text { and } \\
& \mathcal{B}_{(2,-, 2)}^{H y p, C_{2} \times C_{2} \times C_{2}, \theta_{01^{\prime}}} \subset \mathcal{B}(0 ;[2,2] ;\{(2,2)\}) \cap \mathcal{B}_{(2,-, 2)}^{H y p, C_{2} \times C_{2}, \theta_{1^{\prime}}} \cap \mathcal{B}_{(2,-, 2)}^{H y p, C_{2} \times C_{2}, \theta_{0}} \neq \varnothing \\
& \quad \text { Then } \mathcal{B}_{(2,-, 2)}^{K, H y p} \text { is connected. - }
\end{aligned}
$$


Remark 6 Remark that the groups of automorphisms of hyperelliptic nonorientable Klein surfaces with two boundary components and even genus are, as for genus two: $C_{2}, C_{2} \times C_{2}$ and $C_{2} \times C_{2} \times C_{2}$. The subspace of $\mathcal{B}_{(g,-, 2)}^{K, \text { Hyp }}$

provided by NEC groups with signature $\left(0 ;+;\left[2,{ }^{\frac{g}{2}}+\ldots, 2\right],\{(2,2)\}\right)$ is connected and cuts all the other equisymmetric subspaces.

The groups of automorphisms of hyperelliptic non-orientable Klein surfaces with two boundary components of odd genus are: $C_{2}, C_{4}, C_{2} \times C_{2}$ and $D_{4}$. Again, the subspace of $\mathcal{B}_{(g,-, 2)}^{K, \text { Hyp }}$ provided by $N E C$ groups with signature $\left(0 ;+;\left[4,2, \frac{g-1}{2 .}, 2\right],\{(2,2)\}\right)$ (corresponding to surfaces with a unique topological class of actions of $C_{4}$ ) is connected and cuts all the other equisymmetric subspaces.

The hyperelliptic branch loci of non-orientable Klein surfaces with two boundary components are connected.

\section{References}

[AG] Alling, N. L.; Greenleaf, N., Foundations of the Theory of Klein Surfaces. Lecture Notes in Mathematics, Vol. 219. Springer-Verlag, Berlin-New York, 1971. ix +117 pp.

[BCIP] Bartolini G.; Costa A. F.; Izquierdo M., Porto, A. M., On the connectedness of the branch locus of the moduli space of Riemann surfaces. Rev. R. Acad. Cienc. Exactas Fís. Nat. Ser. A Math. RACSAM 104 (2010), no. 1, $81-86$.

[BI] Bartolini, G.; Izquierdo, M., On the connectedness of the branch locus of the moduli space of Riemann surfaces of low genus. Proc. Amer. Math. Soc. 140 (2012), no. 1, 35-45,

[BCI] Bartolini, G.; Costa, A. F.; Izquierdo, M., On the connectivity of branch loci of moduli spaces. Ann. Acad. Sci. Fenn. Math. 38 (2013), 245-258

[BCNS] Bujalance, E.; Costa, A. F.; Natanzon, S. M.; Singerman, D., Involutions of compact Klein surfaces. Math. Z. 211 (1992), no. 3, 461-478.

[BEGG] Bujalance, E.; Etayo, J. J.; Gamboa, J. M.; Gromadzki, G., Automorphism Groups of Compact Bordered Klein Surfaces. A Combinatorial Approach. Lecture Notes in Mathematics, 1439. Springer-Verlag, Berlin, 1990. xiv $+201 \mathrm{pp}$. 
[BEM] Bujalance, E.; Etayo, J. J.; Martínez, E.; Szepietowski, B., On the connectedness of the branch loci of nonorientable unbordered Klein surfaces of low genus. Glasgow Math. J. 57 (2015), 211-230.

[Ci] Cirre, F. J., The moduli space of real algebraic curves of genus 2. Pacific J. Math. 208 (2003), no. 1, 53-72.

[CG] Cirre, F. J.; Gamboa, J. M. Compact Klein surfaces and real algebraic curves. Topics on Riemann Surfaces and Fuchsian Groups (Madrid, 1998), 113-131, London Math. Soc. Lecture Note Ser., 287, Cambridge Univ. Press, Cambridge, 2001.

[C] Costa A. F., Classification of the orientation reversing homeomorphisms of finite order of surfaces. Topology Appl. 62 (1995), no. 2, 145-162.

[CI1] Costa, A. F.; Izquierdo, M., On the connectedness of the locus of real Riemann surfaces. Ann. Acad. Sci. Fenn. Math. 27 (2002), no. 2, 341-356.

[CI2] Costa A. F.; Izquierdo M., On the connectedness of the branch locus of the moduli space of Riemann surfaces of genus 4. Glasg. Math. J. 52 (2010) no. 2, 401-408.

[CIP] Costa A. F.; Izquierdo M., Porto, A. M., On the connectedness of the branch loci of the moduli space of orientable Klein surfaces. Geometriae Dedicata (2014), doi: 10.1007/s10711-014-9983-1

[G] Gamboa, J. M., Compact Klein Surfaces with Boundary Viewed as Real Compact Smooth Algebraic Curves. Mem. Real Acad. Cienc. Exact. Fís. Natur. Madrid 27 (1991), iv+96 pp.

[K] F. Klein, Über Realitätverhältnisse bei der einem beliebigen Geschlechte zugehörigen Normalkurve der $\varphi$. Math. Ann. 42 (1892) 1-29.

[M] Macbeath, A. M., The classification of non-Euclidean plane crystallographic groups, Can. J. Math. 19 (1966) 1192-1205.

[MS] Macbeath, A. M.; Singerman, D., Spaces of subgroups and Teichmüller space. Proc. London Math. Soc. (3) 31 (1975), no. 2, 211-256.

[N1] Natanzon, S. M., Topological classification of pairs of commuting antiholomorphic involutions of Riemann surfaces, Russ. Math. Surv. 45 (1986) 159160.

[N2] Natanzon, S. M., Klein surfaces, Russian Math. Surveys 45 (1990) no. 6, 53-108. 
[N3] Natanzon, S. M., Moduli of Riemann Surfaces, Real Algebraic Curves, and Their Superanalogs. Translated from the 2003 Russian edition by Sergei Lando. Translations of Mathematical Monographs, 225. American Mathematical Society, Providence, RI, 2004. viii+160 pp. ISBN: 0-8218-3594-7.

[Se] Seppälä, M., Real algebraic curves in the moduli space of complex curves. Compositio Math. 74 (1990), no. 3, 259-283.

[Si] Singerman, D., On the structure of non-euclidean crystallographic groups, Proc. Camb. Phil. Soc. 76 (1974) 233-240.

[W] Wilkie, H. C., On non-Euclidean crystallographic groups, Math. Z. 91 (1966) 87-102.

[Y] Yocoyama, K., Complete classification of periodic maps on compact surfaces, Tokyo J. Math. 15 (1992) 247-279. 